EPJ manuscript No.

(will be inserted by the editor)

\title{
Dissipative decoherence in the Grover algorithm
}

\author{
O. V. Zhirov ${ }^{1}$ and D. L. Shepelyansky² \\ 1 Budker Institute of Nuclear Physics, 630090 Novosibirsk, Russia \\ ' 2 Laboratoire de Physique Théorique, UMR 5152 du CNRS, Univ. P. Sabatier, 31062 Toulouse Cedex 4, France
}

November 2, 2005

\begin{abstract}
Using the methods of quantum trajectories we study effects of dissipative decoherence on the accuracy of the Grover quantum search algorithm. The dependence on the number of qubits and dissipation rate are determined and tested numerically with up to 16 qubits. As a result, our numerical and analytical studies give the universal law for decay of fidelity and probability of searched state which are induced by dissipative decoherence effects. This law is in agreement with the results obtained previously for quantum chaos algorithms.
\end{abstract}

PACS. 03.67.Lx Quantum Computation - 03.65.Yz Decoherence; open systems; quantum statistical methods - 24.10.Cn Many-body theory

Nowadays the quantum computing attracts a great interest of the scientific community [1. The main reason of that is due to the fact that certain quantum algorithms allow to perform computations much faster than the usual classical algorithms. The famous example is the Shor algo' rithm which performs factorization of integers on a quantum computer exponentially faster than any known classical factorization algorithm [2]. However, at present there is no mathematical prove about efficiency of potentially possible classical algorithms that in this case gives cer' tain restrictions on the comparative efficiency of classical and quantum algorithms. The situation is different in the case of the Grover quantum search algorithm 3. Indeed, it has been proved that it is quadratically faster than any classical algorithm (see e.g. 11 and Refs. therein).

In addition to the question of quantum algorithm efficiency it is also important to know what is the accuracy of a quantum algorithm in presence of realistic errors and imperfections. The accuracy can be characterized by the fidelity $f$ [4 defined as a scalar product of the wave function of an ideal quantum algorithm and the wave function given by a realistic algorithm (see e.g. 1). In general it is possible to distinguish three types (classes) of errors. The first class can be viewed as random unitary errors in rotational angles of quantum gates. This is the mostly studied case which has been also analyzed for various quantum algorithms with the help of numerical simulations with up to 28 qubits (see e.g. [5,6,7,8,9,10]). It has been shown that in such a case the fidelity decays exponentially with the number of quantum gates $n_{g}$ and with a rate $\gamma$ which is proportional to a mean square of fluctuations in gate rotations. The second class of errors is related to static imperfections (static in time). They are produced by static residual couplings between qubits and static energy shifts of individual qubits which may generate many-body quantum chaos in a quantum computer hardware [1]. This second type of errors, e.g. static imperfections, gives a more rapid decay of fidelity as it has been shown in 12 10. In the case of quantum algorithms for a complex dynamic these imperfections lead to the fidelity decrease described by a universal decay law given by the random matrix theory [10. The two former classes are related to unitary errors. However, there is also the third class which corresponds to the case of nonunitary errors typical to the case of dissipative decoherence. This type of errors has been studied recently for the quantum baker map [13] and the quantum sawtooth map [14. It has been shown that the exponential decay rate of fidelity is proportional to the number of qubits. The dissipative decoherence is treated in the frame of Lindblad equation for the density matrix [15]. A relatively large number of qubits can be reached by using the methods of quantum trajectories developed in [16, 17, 18, 19, 20, 21, 22].

The quantum algorithms studied in $7,8,9,10,12,13$ 14 describe quantum and classical evolution of dynamical systems. However, it is also important to analyze the accuracy of realistic quantum computations for more standard algorithms, e.g. for the Grover algorithm. The effects of unitary errors of the first and second classes have been studied in 23 and 24 respectively. It has been shown that the accuracy of computation is qualitatively different in case of random errors in gates rotations [23] and in the case of static imperfections 24]. Thus it is important to analyze the effects of dissipative decoherence in the Grover algorithm to have a complete picture for this well known algorithm. For that aim we use the approach developed in [13. 14. 
To study the effects of dissipative decoherence in the Grover algorithm we use the same notations as in [24]. Thus, the computational basis of a quantum register with $N=2^{n_{q}}$ states $(\{|x\rangle\}, x=0, \ldots, N-1)$ is used for the algorithm itself. According to [1,3], the initial state $\left|\psi_{0}\right\rangle=$ $\sum_{x=0}^{N-1}|x\rangle / \sqrt{N}$, is transfered to the state

$$
\begin{aligned}
& |\psi(t)\rangle=\hat{G}^{t}\left|\psi_{0}\right\rangle \\
& =\sin \left((t+1 / 2) \omega_{G}\right)|\tau\rangle+\cos \left((t+1 / 2) \omega_{G}\right)|\eta\rangle,
\end{aligned}
$$

after $t$ applications of the Grover operator $\hat{G}$. Here, $\omega_{G}=$ $2 \arcsin (\sqrt{1 / N})$ is the Grover frequency, $|\tau\rangle$ is the search state and $|\eta\rangle=\sum_{x \neq \tau}^{(0 \leq x<N)}|x\rangle / \sqrt{N-1}$. Hence, the ideal algorithm gives a rotation in the $2 \mathrm{D}$ plane $(|\tau\rangle,|\eta\rangle)$. One iteration of the algorithm is given by the Grover operator $\hat{G}$ and can be implemented in $n_{g}=12 n_{\text {tot }}-42$ elementary gates including one-qubit rotations, control-NOT and Toffolli gates as described in 24. The implementation of all these gates requires an ancilla qubit so that the total number of qubits is $n_{t o t}=n_{q}+1$.

To study the effects of dissipative decoherence on the accuracy of the Grover algorithm we follow the approach used in 13 14]. The evolution of the density operator $\rho(t)$ of open system under weak Markovian noises is given by the master equation with Lindblad operators $L_{m}(m=$ $\left.1, \cdots, n_{\text {tot }}\right)$ [15]:

$$
\dot{\rho}=-\frac{i}{\hbar}\left[H_{e f f} \rho-\rho H_{e f f}^{\dagger}\right]+\sum_{m} L_{m} \rho L_{m}^{\dagger},
$$

where the Grover Hamiltonian $H_{G}\left(\hat{G}=\exp \left(-i H_{G}\right)\right)$ is related to the effective Hamiltonian $H_{\text {eff }} \equiv H_{G}-$ $i \hbar / 2 \sum_{m} L_{m}^{\dagger} L_{m}$ and $m$ marks the qubit number. In this paper we assume that the system is coupled to the environment through an amplitude damping channel with $L_{m}=\hat{a}_{m} \sqrt{\Gamma}$, where $\hat{a}_{m}$ is the destruction operator for $m$-th qubit and the dimensionless rate $\Gamma$ gives the decay rate for each qubit per one quantum gate. The rate $\Gamma$ is the same for all qubits.

This evolution of $\rho$ can be efficiently simulated by averaging over the $M$ quantum trajectories (see e.g. 16, [17, 18, 19, 20 21 22 ) which evolve according to the following stochastic differential equation for states $\left|\psi^{\alpha}\right\rangle(\alpha=$ $1, \cdots, M)$ :

$$
\begin{aligned}
& \left|d \psi^{\alpha}\right\rangle=-i H_{s}\left|\psi^{\alpha}\right\rangle d t+\frac{1}{2} \sum_{m}\left(\left\langle L_{m}^{\dagger} L_{m}\right\rangle_{\psi}\right. \\
& \left.-L_{m}^{\dagger} L_{m}\right)\left|\psi^{\alpha}\right\rangle d t+\sum_{m}\left(\frac{L_{m}}{\sqrt{\left\langle L_{m}^{\dagger} L_{m}\right\rangle_{\psi}}}-1\right)\left|\psi^{\alpha}\right\rangle d N_{m},
\end{aligned}
$$

where \langle\rangle$_{\psi}$ represents an expectation value on $\left|\psi^{\alpha}\right\rangle$ and $d N_{m}$ are stochastic differential variables defined in the same way as in [13] (see Eq.(10) there). The above equation can be solved numerically by the quantum Monte Carlo (MC) methods by letting the state $\left|\psi^{\alpha}\right\rangle$ jump to one of $\left.L_{m}\left|\psi^{\alpha}\right\rangle /\left|L_{m}\right| \psi^{\alpha}\right\rangle \mid$ states with probability $\left.d p_{m} \equiv\left|L_{m}\right| \psi^{\alpha}\right\rangle\left.\right|^{2} d t[13]$ or evolve to $\left(1-i H_{e f f} d t / \hbar\right)\left|\psi^{\alpha}\right\rangle / \sqrt{1-\sum_{m} d p_{m}}$ with probability $1-$ $\sum_{m} d p_{m}$. Then, the density matrix can be approximately

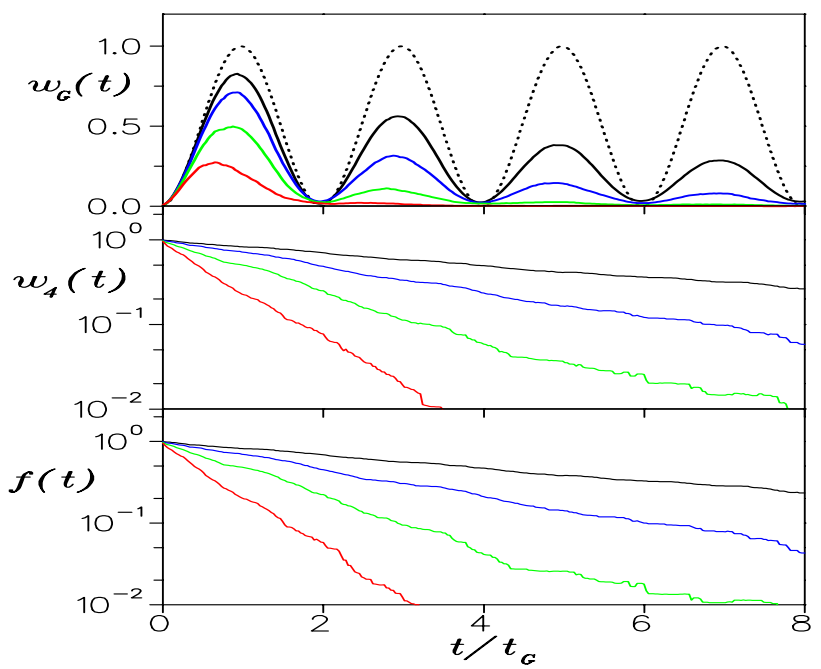

Fig. 1. (color online) Probability of a searched state $w_{G}(t)$ (top), the weight of 4 -state subspace $w_{4}(t)$ (middle) and the fidelity $f(t)$ (bottom) in the Grover algorithm as a function of the iteration step $t$ for $n_{\text {tot }}=12$ qubits, $t_{G}=35.5$. Dotted curve shows data for the ideal Grover algorithm, and solid curves from top to bottom correspond to one qubit decoherence rate $\Gamma=(1,2,4,8) \times 10^{-5}$. Number of quantum trajectories used in simulations is $M=400$.

expressed as

$$
\rho(t) \approx\langle\mid \psi(t)\rangle\langle\psi(t) \mid\rangle_{M}=\frac{1}{M} \sum_{\alpha=1}^{M}\left|\psi^{\alpha}(t)\right\rangle\left\langle\psi^{\alpha}(t)\right|,
$$

where \langle\rangle$_{M}$ represents an ensemble average over $M$ quantum trajectories $\left|\psi^{\alpha}(t)\right\rangle$. Hence, an expectation value of an operator $O$ is given by $\langle O\rangle=\operatorname{Tr}(O \rho) \approx\langle O\rangle_{M}$.

In presence of dissipative decoherence the fidelity $f$ of quantum algorithm is defined as

$$
f(t) \equiv\left\langle\psi_{0}(t)|\rho(t)| \psi_{0}(t)\right\rangle \approx \frac{1}{M} \sum_{\alpha}\left|\left\langle\psi_{0}(t) \mid \psi_{\Gamma}^{\alpha}(t)\right\rangle\right|^{2},
$$

where $\left|\psi_{0}(t)\right\rangle$ is the wave function given by the exact algorithm and $\rho(t)$ is the density matrix of the quantum computer in presence of decoherence, both are taken after $t$ map iterations. Here, $\rho(t)$ is expressed approximately through the sum over quantum trajectories (see also [13).

The effects of dissipative decoherence for the Grover algorithm with $n_{\text {tot }}=12$ qubits are presented in Fig. 1 The data for the probability of the searched state $w_{G}(t)$ clearly show that the Grover oscillations, which have the period $2 t_{G} \approx \pi / \omega_{G}$, start to decay with the number of iterations $t$ due to decoherence. It is not so easy to extract an exponential decay superimposed with oscillations. Therefore, it is convenient to study also the decay of the 4-states probability $w_{4}(t)(|\tau\rangle$ and $|\eta\rangle$ for two states of ancilla qubit, see [24). This probability has a pure exponential decay with the rate $\gamma: w_{4}(t)=\exp (-\gamma t)$. The fidelity decay has the same rate $\gamma$ (see Fig. 11).

It is interesting to note that the decay of Grover oscillations depends on the binary representation of searched 


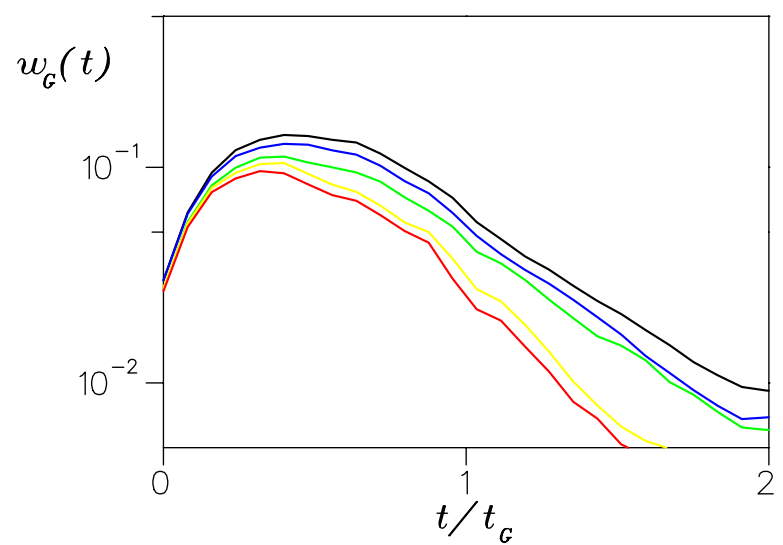

Fig. 2. (color online) Probability of a searched state $w_{G}(t)$ in the Grover algorithm as a function of the iteration step $t$ for $n_{\text {tot }}=9$ qubits. Solid curves from top to bottom correspond to different numbers of units in the binary expansion of the number of a searched state, $n_{u}=1,2,4,6,8$. Number of quantum trajectories used in simulations is $M=2000$.
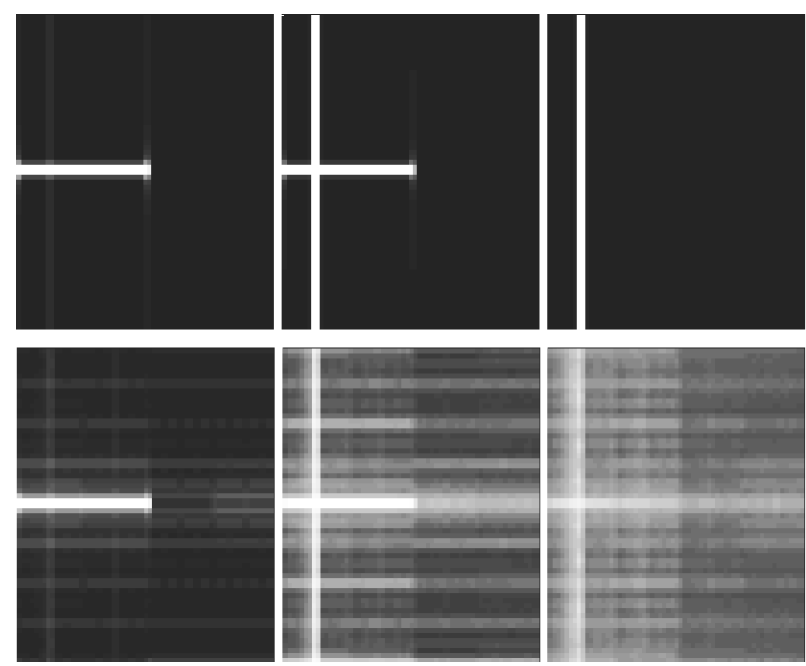

Fig. 3. Husimi function in the Grover algorithm at different number of iterations: $t=1,17,35$, from left to right. Top panels correspond to the ideal Grover algorithm, and bottom panels correspond to the single-qubit decay rate $\Gamma=2 \cdot 10^{-4}\left(n_{\text {tot }}=\right.$ 12). The horizontal axis shows the computational basis $x=$ $0, \ldots, 2 N-1$, while the vertical axis represents the conjugated momentum basis. Density is proportional to grayness changing from maximum (white) to zero (black).

state $|\tau\rangle$. Indeed, the larger is the number $n_{u}$ of spin-up states (numer of units) in the binary representation of $|\tau\rangle$ the faster is the decay of oscillations as it is shown in Fig. 22 The physical origin of this effect is quit clear since in the amplitude decoherence channel the decay $\Gamma$ takes place only for spin-up qubits. However, even if this effect is clearly seen in Fig. 2 in average it is not very strong since during evolution the qubit rotates between two states that leads to averaging of this effect. In the following we will neglect the small deviations produced by this effect and will analyze the averaged behaviour.

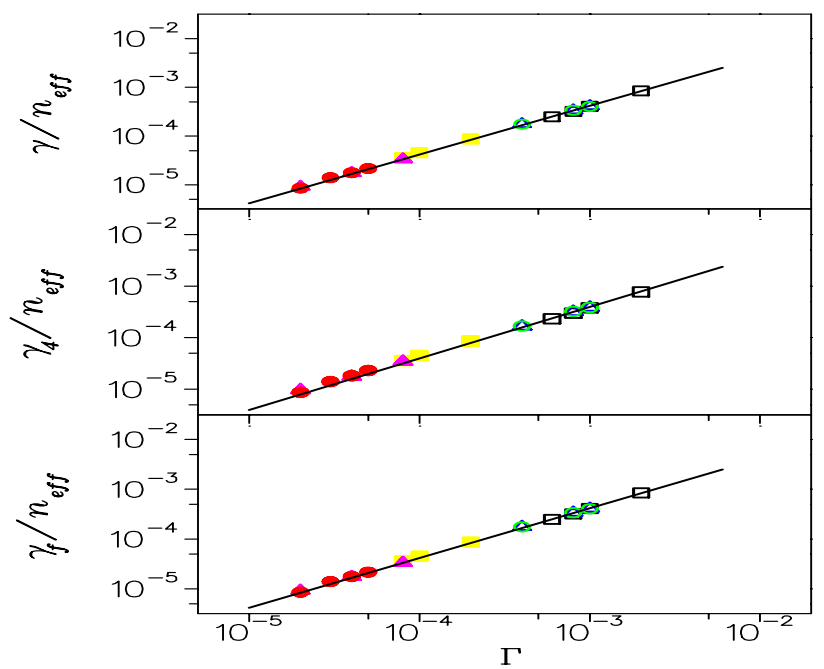

Fig. 4. (color online) Effective rate $\gamma$ for decay of the probability of searched state (top), effective rate $\gamma_{4}$ for decay of the weight of 4-state invariant Grover subspace (middle) and effective fidelity decay rate $\gamma_{f}$ (bottom) as a function of a single qubit decay rate $\Gamma$; $n_{\text {eff }}=n_{g} n_{\text {tot }}$. Data correspond to $n_{\text {tot }}=8$ (open squares), 9 (open triangles), 10 (open circles) and to $n_{\text {tot }}=12$ (full squares), 15 (full triangles), 16 (full circles). Full lines show the average dependence (6).

A pictorial image of the algorithm accuracy can be obtained with a help of the Husimi function [25, 24] which is shown in Fig. 3. In the ideal algorithm the total probability is transfered from the initial state (horizontal white half line corresponding to one state of ancilla qubit) to the searched state (vertical white line) after 35 iterations. In presence of dissipative decoherence the probability of searched state is significantly reduced and probability is transfered to the state $|\eta\rangle$ and many other states of the computational basis (Fig. B3). It is interesting to note that these transitions give homogeneous distribution in the computational basis (at one state of ancilla qubit) and select specific momentum states in the momentum representation that mainly corresponds to dissipative flips of qubits to zero state.

From the data similar to those of Fig. 11 it is possible to obtain the exponential decay law for $w_{G}, w_{4}, f$ $(\propto \exp (-\gamma t))$ and find from it the corresponding decay rates $\gamma, \gamma_{4}, \gamma_{f}$. Their dependence on the one qubit decay

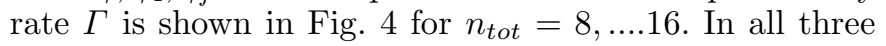
cases the dependence is well described by the relation

$$
\gamma=C \Gamma n_{g} n_{t o t}
$$

where $C \approx 0.4$ is a numerical constant. This result gives the decay rate $\gamma$ which is close to the maximal decay rate $\Gamma_{\max }=\Gamma n_{g} n_{t o t}$ which corresponds to the state with all qubits in the up state. Quite naturally $\gamma$ is proportional to the total number of qubits $n_{t o t}$ since a flip of each qubit from up state gives the decay of fidelity for the whole wave function. The constant $C$ is not sensitive to a variation of the number of qubits that is illustrated in Fig. 5 Indeed, a fit for two groups of qubits $\left(n_{t o t}=8,9,10\right.$ and $n_{t o t}=$ $12,15,16)$ gives close values of $C$ (see Fig. (5). We note that 


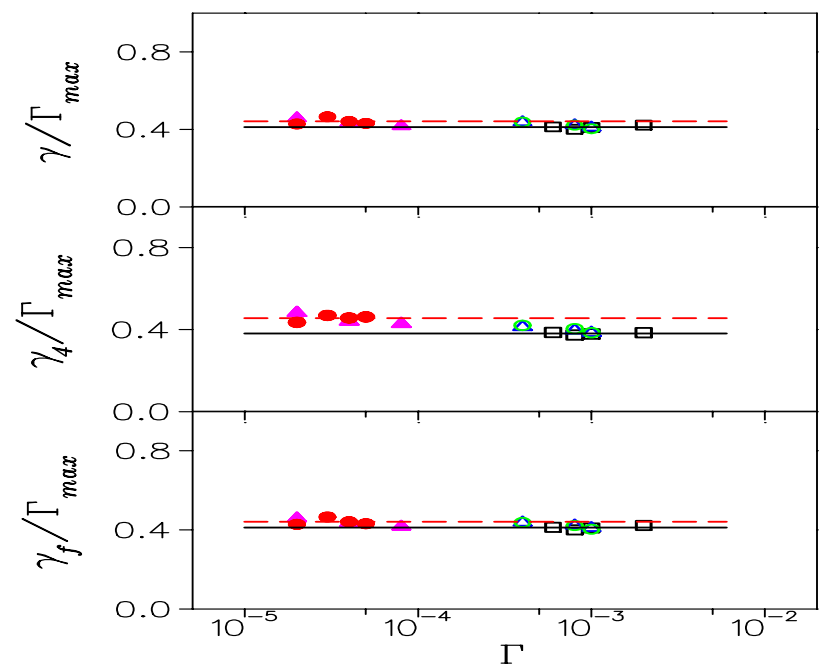

Fig. 5. (color online) Ratios of effective decay rates $\gamma, \gamma_{4}$ and $\gamma_{f}$ (as in Fig 4) to the maximum decay rate $\Gamma_{\max }=\Gamma n_{g} n_{t o t}$ as a function of $\Gamma$. The dashed and full lines give the fit values of constant $C$ obtained for two groups of qubits (dashed for $n_{\text {tot }}=8,9,10$ and full for $\left.n_{\text {tot }}=12,15,16\right)$. The symbols are the same as in Fig 4

$C$ is close to the value $1 / 2$ since in average only a half of qubits is in the up state that reduces the value of $\gamma$ by a factor 2 comparing to the maximum decay rate $\Gamma_{\max }$.

The result (6) is in agreement with the dependence found in 13 14 for other quantum algorithms with dissipative decoherence. This means that the decay rate relation (6) gives a universal description of dissipative decoherence in various quantum algorithms. Therefore it is possible to compare the three classes of quantum errors described at the beginning. The comparison shows that the most rapid decrease of fidelity, and thus the accuracy of quantum computation, is produced by static imperfections. Thus it is necessary to develop specific error correction methods which will be able to handle effects of static imperfections in quantum algorithms. First steps in this direction are done recently in [26,27]28].

This work was supported in part by the EC IST-FET projects EDIQIP and EuroSQIP and (for OVZ) by RAS Joint scientific program "Nonlinear dynamics and solitons".

\section{References}

1. M. A. Nielsen, and I. L. Chuang, Quantum computation and quantum information, Cambridge Univ. Press (2000).

2. P. W. Shor, in Proc. 35th Annu. Symp. Foundations of Computer Science (ed. Goldwasser, S. ), 124 (IEEE Computer Society, Los Alamitos, CA, 1994).

3. L. K. Grover, Phys. Rev. Lett. 79, 325 (1997).

4. A. Peres, Phys. Rev. A 30, 1610 (1984).

5. J. I. Cirac and P. Zoller, Phys. Rev. Lett. 74, 4091 (1995).

6. C. Miquel, J. P. Paz, and W. H. Zurek, Phys. Rev. Lett. 78, 3971 (1997).

7. B.Georgeot and D.L.Shepelyansky, Phys. Rev. Lett. 86, 5393 (2001).
8. M. Terraneo, B. Georgeot and D.L. Shepelyansky, Eur. Phys. J. D 22, 127 (2003).

9. S. Bettelli, Phys. Rev. A 69, 042310 (2004).

10. K.M.Frahm, R.Fleckinger and D.L.Shepelyansky, Eur. Phys. J D 29, 139 (2004).

11. B.Georgeot and D.L.Shepelyansky, Phys. Rev. E 62, 3504 (2000); 62, 6366 (2000).

12. G.Benenti, G.Casati, S.Montangero and D.L.Shepelyansky, Phys. Rev. Lett. 87, 227901 (2001).

13. G. Carlo, G. Benenti, G. Casati and C. Mejia-Monasterio, Phys. Rev. A 69, 062317 (2004).

14. J.W. Lee and D.L. Shepelyansky, Phys. Rev. E 71, 056202 (2005).

15. G. Lindblad, Commun. Math. Phys. 48, 119 (1976); V. Gorini, A. Kossakowski, and E.C.G. Sudarshan, J. Math. Phys. 17, 821 (1976).

16. H.J. Carmichel, An Open Systems Approach to Quantum Optics (Springer, Berlin, 1993).

17. R. Dum, P. Zoller, and H. Ritsch, Phys. Rev. A 45, 4879 (1992).

18. J. Dalibard, Y. Castin, and K. Mølmer, Phys. Rev. Lett. 68, 580 (1992).

19. N. Gisin, Phys. Rev. Lett. 52, 1657 (1984).

20. R. Schack, T.A. Brun, and I.C. Percival, J. Phys. A 28, 5401 (1995).

21. A. Barenco, T.A. Brun, R. Schack, and T.P. Spiller, Phys. Rev. A 56, 1177 (1997).

22. T.A. Brun, Am. J. Phys. 70, 719 (2002); quant-ph/0301046

23. P.H. Song and I. Kim, Eur. Phys. J. D 23, 299 (2003).

24. A.A. Pomeransky, O.V.Zhirov and D.L. Shepelaynsky, Eur. Phys. J. D 31, 131 (2004).

25. S.-J. Chang and K.-J. Shi, Phys. Rev. A 34, 7 (1986).

26. O.Kern, G.Alber and D.L.Shepelyansky, Eur. Phys. J. D 32, 153 (2005).

27. A.A.Pomeransky, O.V.Zhirov and D.L.Shepelyansky, quant-ph/0407264

28. O.Kern, and G.Alber, quant-ph/0506038 